\title{
Diagnosis and management of ascites and hepatorenal syndrome (acute kidney injury) in cirrhosis
}

- Brief author biography for all authors - including qualifications, current position and location, and research interest

\author{
Alastair O'Brien MBBS, BSc, PhD, FRCP
}

Reader in Experimental Hepatology and Consultant Hepatologist, Institute of Liver Disease and Digestive Health, University College London, University College \& The Royal Free Hospitals

Bio: My research team is dedicated to improving management of infection in advanced liver disease. We use clinical trial, laboratory molecular studies and analysis of large data sets to achieve this goal. I have received generous funding from the Wellcome Trust/DoH, NIHR, MRC, BBSRC, British Society of Gastroenterology and Rosetrees Trust.

\section{- Declaration of competing interests}

Competing interests: none declared'

\section{- Abstract (no more than $\mathbf{2 0 0}$ words)}

Ascites is the most common complication of cirrhosis, and represents a watershed moment with patients' median survival falling from greater than 12 years for compensated cirrhosis to approximately two years. Treatment aims to reduce sodium intake and increasing renal sodium excretion with the aldosterone antagonist, spironolactone, starting at $100 \mathrm{mg}$ daily and $60 \%$ respond. Renal dysfunction affects as many as $20 \%$ of hospitalized patients, and is a strong predictor of mortality. Liver Transplantation represents the best treatment for Hepatorenal syndrome (HRS) yet is rarely available and the combination of vasoconstrictors and albumin represent the mainstay of treatment. Terlipressin is the most widely utilised vasoconstrictor leading to HRS resolution in over $50 \%$. The role of Transjugular intrahepatic portosystemic shunt insertion or renal replacement therapy for HRS remains uncertain with further studies needed. These patients are very fragile with a high mortality. Many patients require regular ascitic drainage every 2-4 weeks that is best achieved in a day case setting. Diuretic medication requires frequent dose-adjustment due to co-existent renal impairment. In those that ascites persists, liver transplantation or TIPPS should be considered. Finally, given the poor prognosis for many it is important to address end of life planning and palliation where appropriate.

\section{- 5-10 keywords}

Terlipressin, Diuretics, Albumin, Infection, Splanchnic vasodilatation, Systemic inflammation

\section{- Contact details for each author, including postal and email address}

Institute for Liver and Digestive Health' Division of Medicine, $3^{\text {rd }}$ Floor, Royal Free Campus, University College London, London, United Kingdom; rmhaajo@ucl.ac.uk

- Word count (excluding abstract and references)

2723

- Number of figures and tables

1 Table

\section{- Self-assessment questions}


How would you investigate and treat a patient presenting for the first time with ascites?

A patient with known alcohol-induced cirrhosis presents to A\&E with a creatinine of $175 \mathrm{mmol} / \mathrm{l}$. How would you investigate and treat him?

This patient makes a successful recovery. How would you subsequently manage him? 


\section{- How common is liver disease?}

Liver disease is increasing in prevalence worldwide with 60000 people in the UK estimated to have cirrhosis. In contrast to other common diseases in the UK, mortality rates have increased $400 \%$ since 1970 and it is now the third commonest cause of premature death. Indeed liver disease is predicted to shortly overtake ischaemic heart disease as the leading cause of working life years lost in the UK. Alcohol remains the primary driver for the vast majority of cirrhosis-related hospital admissions with a median age of presentation in the early 50 s but obesity is rapidly rising and non-alcoholic steatohepatitis will continue to maintain the increasing liver disease mortality. There have been great successes, however, with the extraordinary effectiveness of the oral antiviral regimens against Hepatitis C virus (HCV) leading to campaigns to identify and treat all patients with HCV.

Ascites is the most common complication of cirrhosis or decompensation event, with $5 \%$ to $10 \%$ of uncomplicated (compensated) liver cirrhosis patients per year developing this. Decompensation represents a watershed moment for patients with cirrhosis, with the median survival falling from greater than 12 years for compensated cirrhosis to approximately two years.

\section{- Definitions}

Ascites is the accumulation of fluid within the peritoneal cavity with a volume of at least $25 \mathrm{ml}$, although greater than 14 litres may collect. Over $75 \%$ are due to liver cirrhosis and portal hypertension. Other causes include pancreatitis, malignancy or tuberculosis.

Renal dysfunction in those with ascites is common, affecting as many as $20 \%$ of hospitalized patients, and is a strong predictor of mortality. Renal dysfunction in cirrhosis has been defined as a serum creatinine $(\mathrm{sCr})>133 \mu \mathrm{mol} / \mathrm{L}(1.5 \mathrm{mg} / \mathrm{dl})$ and acute kidney injury (AKI) as a $50 \%$ increase of $\mathrm{s} C \mathrm{r}$ to $>133$ $\mu \mathrm{mol} / \mathrm{L}^{1}$. The main differential diagnoses are hepatorenal syndrome (HRS) and acute tubular necrosis (ATN); if there has been no recent use of nephrotoxic drugs, no hematuria, no significant proteinuria, no shock and no structural renal abnormalities, the diagnosis is HRS. Classically HRS was considered a functional renal failure caused by intrarenal vasoconstriction resulting from arterial vasodilation and arterial hypotension. For many years type $1 \mathrm{HRS}$ was defined as rapidly progressive renal failure, with a doubling of $\mathrm{sCr}$ to $>221 \mu \mathrm{mol} / \mathrm{L}(2.5 \mathrm{mg} / \mathrm{dL})$ or a halving of the creatinine clearance to $<20 \mathrm{~mL} / \mathrm{min}$ in less than two weeks. Type $2 \mathrm{HRS}$ is slower in onset, not associated with an obvious precipitant and classically defined by an increase in $\mathrm{sCr}$ to $>133 \mu \mathrm{mol} / \mathrm{L}(1.5 \mathrm{mg} / \mathrm{dL})$ or reduction in creatinine clearance to $<40 \mathrm{~mL} / \mathrm{min}$ and a urine sodium $<10 \mu \mathrm{mol} / \mathrm{L}$. Most patients that go on to develop type 2 HRS have diuretic-resistant ascites beforehand, in which the kidneys are unable to excrete sufficient sodium to clear ascites despite diuretic medication.

More recently, HRS has been recognised as consequent to haemodynamic and inflammatory changes and that AKI may occur in those with underlying chronic kidney disease. Therefore, in the revised classification, type 1 HRS corresponds to HRS-AKI and type 2 renal impairment in those that fulfil criteria of HRS but not AKI.

\section{- Epidemiology}

The aetiology of liver disease differs according to geographical location. In the developed world, it is most often due to obesity - non-alcoholic fatty liver disease (NAFLD) or non-alcoholic steatohepatitis (NASH), and alcohol excess. In the developing world, chronic viral infection with either hepatitis B or hepatitis $C$ is the leading cause of liver mortality. Globally, about half a billion people suffer from chronic viral hepatitis.

\section{- Pathology and pathogenesis}


Ascites: Splanchnic arterial vasodilation is the major underlying cause for increased water and sodium retention in patients with cirrhosis and ascites. Portal hypertension (defined as a hepatic venous pressure gradient (HVPG) measurement $>5 \mathrm{~mm} \mathrm{Hg}$ and clinically significant once HVPG $>10-12 \mathrm{~mm}$ $\mathrm{Hg}$ ) causes the release of vasodilators (e.g. nitric oxide (NO) and carbon monoxide (CO)) in the splanchnic circulation. This reduces the effective circulating volume (ECV), activating baroreceptors and the sympathetic nervous system (SNS), renin-angiotensin-aldosterone system (RAAS) and nonosmotic secretion of vasopressin. Cardiac output increases to compensate for the reduced ECV but as liver disease progresses, the heart is unable to cope with further reduction in ECV and subsequent activation of the RAAS increases renal reabsorption of sodium and water, leading to the appearance of ascites. The hyperactivation of the SNS triggers an increase in proximal tubular absorption of sodium and water, further increasing ascites. Pathological translocation of bacteria or bacterial products from the gut to the systemic circulation may also contribute by stimulating release of proinflammatory cytokines leading to additional release of $\mathrm{NO}$ and $\mathrm{CO}$, increasing splanchnic arterial vasodilation even more.

Hepatorenal Syndrome: Pathophysiology involves both hypoperfusion leading to renovascular dysfunction and systemic inflammation caused by bacterial translocation. Increased pro-inflammatory cytokines production is associated with renal dysfunction in spontaneous bacterial peritonitis (SBP) and acute-on-chronic liver failure (ACLF) ${ }^{3}$. These processes may drive mitochondria-mediated metabolic down-regulation of the kidneys. In patients with severe cholestasis there may be additional renal tubular injury from excessive bile salts.

\section{- Course of the disease}

The natural history of cirrhosis is characterised by an asymptomatic course until increasing portal pressure and worsening liver function lead to clinical signs. Compensated cirrhosis patients generally have a good quality of life and the disease may remain undetected for many years. Decompensation is characterised by the development of ascites, gastrointestinal (GI) bleeding, encephalopathy, and jaundice. This marks a significant change in disease trajectory, often with rapid progression that is worsened by increased incidence of bacterial infections which, when complicated by AKI, with or without HRS, have a particularly poor prognosis.

\section{- Diagnosis}

Abdominal swelling may develop over days or several weeks. The presence of fluid is confirmed clinically by demonstrating shifting dullness. Precipitating factors include continued excessive alcohol use, a high-sodium diet, infection, development of a hepatocellular carcinoma or splanchnic vein thrombosis. Mild abdominal pain and discomfort are common but, if more severe, should raise the suspicion of spontaneous bacterial peritonitis (SBP). Respiratory distress and difficulty eating accompany tense ascites. Patients may also have non-specific symptoms, particularly weakness, anorexia and fatigue. Other specific symptoms include ${ }^{2}$ :

- Peripheral oedema

- Pleural effusion (usually right-sided) may infrequently be found and arises from the passage of ascites through congenital diaphragmatic defects

- Haematemesis and melaena from gastrointestinal haemorrhage

- Pruritus due to cholestasis

- Gynaecomastia, loss of libido and amenorrhoea due to endocrine dysfunction

- Confusion and drowsiness due to neuropsychiatric complications (portosystemic hepatic encephalopathy). 
- Investigations and differential diagnoses

A diagnostic aspiration of $10-20 \mathrm{~mL}$ of fluid is mandatory to test for:

- Cell count: a neutrophil count $>250$ cells $/ \mathrm{mm}^{3}$ is diagnostic of an underlying (usually spontaneous) bacterial peritonitis.

- Gram stain and culture.

- Protein measurement: a high serum-ascites albumin gradient of $>11 \mathrm{~g} / \mathrm{L}$ suggests portal hypertension, and a low gradient $<11 \mathrm{~g} / \mathrm{L}$ is associated with non-liver disease-related abnormalities of the peritoneum, such as neoplasia (Table 1 ).

- Cytology: to search for malignant cells.

- Amylase: to exclude pancreatic ascites.

Liver and Renal tract ultrasound: to examine liver parenchyma, vascularity and exclude renal tract obstruction

As many as $25 \%$ may have a cause other than cirrhosis and the combination of ascitic fluid (AF) protein count, liver blood tests and abdominal imaging is able to differentiate in the majority (see table 1). In some cases, liver cirrhosis may co-exist with other causes such as metastatic malignancy. Patients may also have intrinsic renal disease with or without HRS-AKI and this will become more common as NASH increases in prevalence with patients also affected by hypertension and type 2 diabetes.

Two prospective studies comprising greater than 100 patients each confirmed low ascitic fluid (AF) protein concentration as an independent predictor of spontaneous bacterial peritonitis (SBP), a dreaded complication of ascites ${ }^{1}$. AF protein content is believed to mirror host opsonisation activity with SBP incidence rates within 2 years of sampling of $20-25 \%$ at levels $<1 \mathrm{~g} / \mathrm{dL}$ and $<1 \%$ in patients with protein levels $>1.5 \mathrm{~g} / \mathrm{dL}$. These data have been challenged by two recent post-hoc analyses of three large cohorts of hospitalised patients with decompensated cirrhosis that suggested ascitic protein count did not correlate with SBP risk. A large-scale clinical trial will shortly commence in the UK to determine if primary antibiotic prophylaxis with co-trimoxazole can prevent SBP in patients with a low AF protein count.

\section{- Management}

\section{Ascites}

It is important to consider the underlying cause of cirrhosis. Administration of the new direct acting anti-hepatitis $\mathrm{C}$ agents may lead to ascites resolution, as will greater than 6 months alcohol cessation in a significant number of patients if this is the primary driver. We await effective treatments for NASH. Otherwise, treatment is aimed at reducing sodium intake and increasing renal sodium excretion, producing a net reabsorption of fluid from the ascites into the circulating volume. The maximum rate at which ascites can be mobilized is $500-700 \mathrm{~mL}$ in 24 hours. It is possible to reduce sodium intake to $40 \mathrm{mmol}$ in $24 \mathrm{~h}$ and maintain a palatable diet with adequate protein and calorie intake. Many drugs contain significant amounts of sodium (up to $50 \mathrm{mmol}$ daily) especially penicillins and cephalosporins and sodium-retaining drugs such as NSAIDs or corticosteroids should be avoided. Fluid restriction is unnecessary.

The diuretic of choice is the aldosterone antagonist, spironolactone, starting at $100 \mathrm{mg}$ daily. $60 \%$ respond on this regimen, but the spironolactone can be increased gradually to $400 \mathrm{mg}$ daily if necessary, providing there is no hyperkalaemia. Urinary sodium can be used to titrate the dose. The loop diuretic, furosemide $40 \mathrm{mg}$ is added if response is poor but has several potential disadvantages, including hyponatraemia, hypokalaemia and volume depletion. Chronic administration of 
spironolactone may cause produce gynaecomastia and amiloride should be substituted at $5-10 \mathrm{mg}$ a day. Diuretics should be temporarily discontinued in the event of hyponatraemia (sodium $<128 \mathrm{mmol} / \mathrm{L}$ ) a rise in serum creatinine, hyperkalaemia or worsening encephalopathy. Vaptans, vasopressin $\mathrm{V}_{2}$-receptor antagonists that increase free water clearance, have a small beneficial effect on hyponatraemia and ascites but do not affect mortality, complications of cirrhosis or renal failure and routine use in cirrhosis cannot be recommended.

Paracentesis (drainage of ascites) relieves symptomatic tense ascites. The main complications are hypovolaemia and renal dysfunction (post-paracentesis circulatory dysfunction) which is more likely with $>5 \mathrm{~L}$ removal and worse liver function. This is prevented by infusing albumin $(8 \mathrm{~g} / \mathrm{L}$ of ascitic fluid removed). In practice, up to $20 \mathrm{~L}$ can be removed over $4-6$ hours, with albumin infusion.

\section{Renal Dysfunction}

Several precipitating factors for renal dysfunction should be avoided such as nephrotoxic drugs, NSAIDs or diuretic-induced excessive diuresis, or treated rapidly such as infections or GI bleeding. Contrast imaging ought to be performed cautiously in patients with ascites. Finally, the increase in intra-abdominal pressure associated with tense ascites may lead to AKI.

The most common cause of AKI in hospitalised patients with decompensated cirrhosis is pre-renal, accounting for approximately $70 \%$. Intrarenal-AKI is predominantly acute tubular necrosis (ATN) and post-renal AKI is uncommon in cirrhosis. Given most cases of pre-renal AKI are resolved by volume expansion and post-renal AKI is uncommon, the key point is to differentiate HRS-AKI from ATN. However renal biopsy is rarely performed and the distinction between HRS-AKI and ATN is difficult. Recently, novel biomarkers have emerged in this setting and urinary neutrophil gelatinase associated lipocalin (NGAL) appears the most promising.

The cause of AKI must be investigated urgently to prevent progression and management commenced immediately. Diuretics and beta-blockers should be discontinued and most importantly volume replacement is required in accordance with the cause and the severity of fluid loss. Patients with diarrhoea or excessive diuresis may be given crystalloids, and those with GI bleeding packed red blood cells to maintain haemoglobin level 7-9 g/dl. In patients with AKI and tense ascites, therapeutic paracentesis associated with albumin infusion improves renal function. If no obvious cause and HRSAKI AKI stage $>1 \mathrm{~A}$ (increase of serum creatinine 2 -fold from baseline) $20 \%$ albumin solution at the dose of $1 \mathrm{~g} / \mathrm{kg}$ of body weight (with a maximum of $100 \mathrm{~g}$ ) for two consecutive days is recommended.

Liver Transplantation represents the best treatment for HRS but is rarely available and the combination of vasoconstrictors and albumin represent the mainstay of treatment. Vasoconstrictors counteract splanchnic arterial vasodilation and albumin the reduction in ECV and improves cardiac contractility with a dose of $20-40 \mathrm{~g} /$ day recommended. Terlipressin is the most widely utilised vasoconstrictor leading to HRS resolution in over $50 \%^{4}$. Alternatives are the alpha-adrenergic drug, midodrine given orally ( 2.5 up to 12.5 qid) with subcutaneous octreotide (125 up to 250 lgr bid) or a continuous intravenous infusion of norepinephrine (0.1-1 mg per hour).

Studies have shown terlipressin to be superior to midodrine plus octreotide and norepinephrine and terlipressin to be equivalent for HRS reversal and 1-month survival. Cochrane analysis demonstrated a beneficial effect on mortality with terlipressin but it increased the risk of serious cardiovascular adverse events. A continuous intravenous infusion of terlipressin was associated with a lower rate of adverse effects than intravenous boluses. Treatment with vasoconstrictors plus albumin should be 
continued until sCr falls to $<133 \mu \mathrm{mol} / \mathrm{L}$. HRS recurs in $20 \%$ following treatment withdrawal, but retreatment is often effective.

Transjugular intrahepatic portosystemic shunt insertion has good theoretical reasons for being an effective treatment for HRS as it reduces portal hypertension and increases cardiac output, improving renal perfusion, sodium and water excretion. In a retrospective, matched cohort analysis TIPS placement was associated with significant improvement in kidney function compared to patients undergoing serial large volume paracenteses. This was most prominent in participants with baseline eGFRs $<60 \mathrm{~mL} / \mathrm{min} / 1.73 \mathrm{~m}$. Although some patients with hepatorenal syndrome can benefit from a TIPS procedure, this must be balanced against the high risk, particularly for development of hepatic encephalopathy.

The limited data available for the role of renal replacement therapy (RRT) in patients with HRS has not demonstrated a survival benefit and this remains contentious because of the grim prognosis for these patients ${ }^{5}$. For patients on the transplant waiting list RRT is an appropriate treatment for volume overload, severe metabolic acidosis or hyperkalemia. Continuous RRT carries a lower risk of hypotension than intermittent hemodialysis but the best RRT method in cirrhosis is unknown. In nonresponders to medical treatment who are candidates for LT, a simultaneous kidney-liver transplantation can be considered if renal dysfunction is thought unlikely to reverse. In patients not eligible for LT the decision for RRT should be made individually and aim to avoid futility.

\section{- Prognosis, explanation to the patient and follow up}

Patients with complicated ascites represent a very fragile population with a high mortality and a high risk of early readmission. Patients with alcohol as the predominant cause need urgent cessation therapy with a realistic opportunity for ascites resolution. Many patients require regular ascitic drainage every 2-4 weeks that is best achieved in a day case setting. Diuretic medication requires frequent dose-adjustment due to co-existent renal impairment. In those that ascites persists liver transplantation or TIPPS should be considered.

A peritoneo-bladder conduit, by means of an implantable, rechargeable, battery-powered pump, has been developed for use in patients with resistant ascites (alfapump ${ }^{\circledR}$ ). This removes ascites from the peritoneal cavity into the urinary bladder, to be eliminated in urine. Early studies have shown a reduction in the need for large-volume paracentesis but several complications, including pain and infection, occur.

Patients require surveillance for development of hepatocellular carcinoma with 6 monthly ultrasonography and screening endoscopy for varices. Finally, given the poor prognosis for many it is important to address end of life planning and consider palliative measures where appropriate.

\section{- Prevention of HRS with Human Albumin Solution}

A large randomised controlled trial in patients admitted to hospital with SBP showed that treatment with $20 \%$ albumin (1.5mg. $\mathrm{kg}^{-1}$ on day 1 and $1 \mathrm{mg} \mathrm{kg}^{-1}$ on day 3 ) prevented HRS-AKI, although the control group received no fluid. A more recent trial of the same regimen of albumin in patients with infections other than SBP was stopped early due to the increased incidence of pulmonary oedema in the treatment group. The recent ANSWER trial of weekly outpatient albumin infusions $(200 \mathrm{ml}$ of $20 \%$ ) compared with standard medical therapy (no fluids) demonstrated a reduced incidence of renal dysfunction and HRS type1 during the 18-month trial period ${ }^{6}$. However weekly outpatient albumin infusions represent a substantial logistical challenge and this approach has not been introduced presently in the UK. 


\section{- Conclusions}

The management of patients with ascites \pm renal dysfunction is complex, currently hospital-based and highly resource-intensive. The development of new models of specialist care that are outpatient based and prevention focused are urgently required to improve the survival and quality of life in this increasing group of patients.

\section{References}

1. Runyon BA \& AASLD. American Association for the Study of Liver Diseases Practice Guideline adult patients with ascites 2012. Hepatology 57, 1651-1653 (2013).

2. Angeli P, Bernardi M, Villanueva C, Francoz C, Mookerjee RP, et al. EASL Clinical Practice Guidelines for the management of patients with decompensated cirrhosis. J Hepatol. 2018 Aug;69(2):406-460. doi: 10.1016/j.jhep.2018.03.024. Epub 2018 Apr 10.

3. Piano S, Tonon M, Angeli P. Management of ascites and hepatorenal syndrome. Hepatol Int. 2018 Feb;12(Suppl 1):122-134. doi: 10.1007/s12072-017-9

4. Boyer TD, Sanyal AJ, Wong F, Frederick RT, Lake JR, et al; REVERSE Study Investigators. Terlipressin Plus Albumin Is More Effective Than Albumin Alone in Improving Renal Function in Patients With Cirrhosis and Hepatorenal Syndrome Type 1. Gastroenterology. 2016 Jun;150(7):15791589.e2. doi: 10.1053/j.gastro.2016.02.026. Epub 2016 Feb 16.

5. O'Brien, A.J., Welch, C.A., Singer, M. \& Harrison, D.A. Prevalence and outcome of cirrhosis patients admitted to UK intensive care: a comparison against dialysis-dependent chronic renal failure patients. Intensive care medicine 38, 991-1000 (2012).

6. Caraceni P, Riggio O, Angeli P, Alessandria C, Neri S, et al; ANSWER Study Investigators. Long-term albumin administration in decompensated cirrhosis (ANSWER): an open-label randomised trial. Lancet. 2018 Jun 16;391(10138):2417-2429. doi: 10.1016/S0140-6736(18)30840-7. 


\section{Table 1}

The serum-ascites albumin gradient

High serum-ascites albumin gradient (>11 $\mathrm{g} / \mathrm{L}$ )

- Portal hypertension, e.g. hepatic cirrhosis

- Hepatic outflow obstruction

- Budd-Chiari syndrome

- Hepatic veno-occlusive disease

- Tricuspid regurgitation

- Constrictive pericarditis

- Right-sided heart failure

Low serum-ascites albumin gradient ( $<11 \mathrm{~g} / \mathrm{L})$

- Peritoneal carcinomatosis

- Peritoneal tuberculosis

- Pancreatitis

- Nephrotic syndrome

(Modified from Chung RT, lafrate AJ, Amrein PC et al. Case records of the Massachusetts General Hospital. New England Journal of Medicine 2006; 354:2166-2175.) 